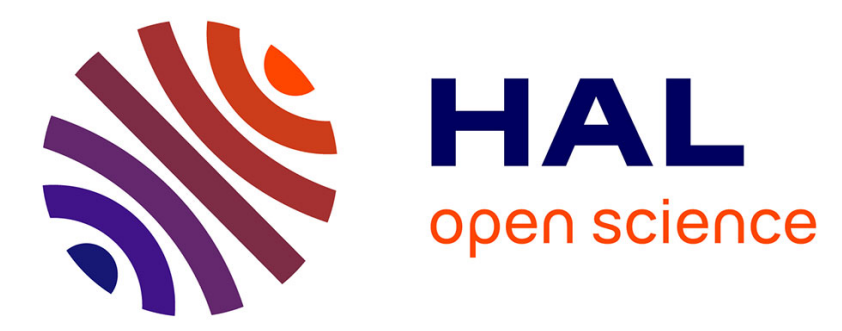

\title{
Energy measurement in nonlinearly coupled nanomechanical modes
}

Alexei Gaidarzhy, Jerome Dorignac, Guiti Zolfagharkhani, Matthias Imboden, Pritiraj Mohanty

\section{- To cite this version:}

Alexei Gaidarzhy, Jerome Dorignac, Guiti Zolfagharkhani, Matthias Imboden, Pritiraj Mohanty. Energy measurement in nonlinearly coupled nanomechanical modes. Applied Physics Letters, 2011, 98, pp.264106. 10.1063/1.3604797 . hal-00628025

\section{HAL Id: hal-00628025 \\ https://hal.science/hal-00628025}

Submitted on 31 May 2021

HAL is a multi-disciplinary open access archive for the deposit and dissemination of scientific research documents, whether they are published or not. The documents may come from teaching and research institutions in France or abroad, or from public or private research centers.
L'archive ouverte pluridisciplinaire HAL, est destinée au dépôt et à la diffusion de documents scientifiques de niveau recherche, publiés ou non, émanant des établissements d'enseignement et de recherche français ou étrangers, des laboratoires publics ou privés. 


\title{
Energy measurement in nonlinearly coupled nanomechanical modes
}

\author{
Alexei Gaidarzhy, ${ }^{1, a)}$ Jérôme Dorignac, ${ }^{2,3}$ Guiti Zolfagharkhani, ${ }^{1}$ Matthias Imboden, ${ }^{4}$ \\ and Pritiraj Mohanty 4 \\ ${ }^{1}$ Sand 9, Inc., One Kendall Square B2305, Cambridge, Massachusetts 02139, USA \\ ${ }^{2}$ Département de Physique Théorique, Laboratoire Charles Coulomb, UMR 5221 CNRS-UM2, \\ Université Montpellier 2 Montpellier, France \\ ${ }^{3}$ Department of Computer and Electrical Engineering, Boston University, 44 Cummington Street, Boston, \\ Massachusetts 02215, USA \\ ${ }^{4}$ Department of Physics, Boston University, 590 Commonwealth Avenue, Boston, Massachusetts 02215, USA
}

(Received 9 May 2011; accepted 6 June 2011; published online 1 July 2011)

\begin{abstract}
We report direct measurements of average vibration energy in a high frequency flexural resonance mode achieved via an-harmonic elastic coupling to a fundamental vibration mode of a nanomechanical resonator. The second order coupling effect produces a frequency shift of the readout mode as a function of the mean square of the excitation amplitude of the high order mode. We measure frequency shifts at the lowest driving amplitudes, down to the noise floor of the experimental setup. With implementation of existing ultra-sensitive amplifiers, the reported technique will enable direct measurements of quantized energy transitions in low-thermal occupation number nanomechanical resonators. (C) 2011 American Institute of Physics. [doi:10.1063/1.3604797]
\end{abstract}

Much experimental effort has been focused on demonstrating quantum mechanical effects in macroscopic mechanical resonators. ${ }^{1-7}$ Access to quantum properties of mechanical systems, such as energy quantization phenomena, ${ }^{8,9}$ can be achieved via nonlinear systems and the coupling to resonator energy, as opposed to position or velocity. Among the proposed methods, Santamore et al. ${ }^{10}$ argue that nonlinearities inherent in modal interactions of a single resonator can be utilized for direct measurements of the energy in a nanomechanical mode. Martin and Zurek $^{2}$ estimate that if in addition the dynamics of the detector are much slower than that of the quantum system, the back-action is reduced as the detector cannot effectively cause transitions between the energy states. In such a setup, a measurement of the phonon number in the quantized system can be performed with sufficient bandwidth and signal-to-noise ratio in order to resolve spontaneous state transitions, which offers the possibility of generating the necessary non-classical Fock or energy-number states and accessing discrete transitions among them.

Here, we describe of a coupled-mode nanomechanical system that provides the experimental realization of energy measurement in a high frequency nanomechanical mode. Our scheme uses nonlinear modal interaction of two widely spaced resonances on a single hybrid nanobeam. The lower frequency mode can serve as a detector of the average energy of the high frequency mode for energy quantization measurements under appropriate conditions. These can include millikelvin temperatures to lower the thermal occupation of the system mode, sufficiently fast measurement times compared to the lifetimes of Fock states to resolve individual transitions, as well as ultra-low noise amplification of the detector signal.

The hybrid coupled-element design of our nanomechanical resonator has been analyzed in previous publications. ${ }^{11-13}$ The resonator's spectral response can be approximated as a

\footnotetext{
${ }^{\text {a) }}$ Author to whom correspondence should be addressed. Electronic mail: alexei@sand9.com.
}

two-mode system, in which the fundamental and collective modes are widely separated in frequency, see Fig. 1. The high frequency collective mode is the system mode, whose average mechanical energy is probed using the low frequency detector mode. The devices used in this experiment were fabricated from nano-crystalline diamond films using e-beam lithography and micro machining techniques. ${ }^{14}$ The resonator devices are placed in direct thermal contact with the mixing chamber of a dilution refrigerator, cooled to $40 \mathrm{mK}$ inside a vacuum-tight metallic chamber at the canter of a $16 \mathrm{~T}$ superconducting solenoid magnet. The resonator modes are excited magnetomotively by passing an RF (radio frequency) current via the metalized resonator electrodes in a magnetic field. ${ }^{15}$ The displacement-induced Faraday voltage is amplified using a cryogenic preamplifier with $0.5 \mathrm{nV} / \sqrt{\mathrm{Hz}}$ input noise at the $77 \mathrm{~K}$ temperature stage. The spectral power of the amplitude modulated signal is measured in a spectrum analyzer.

To probe the excitation level of the high frequency mode, we measure the small changes in the resonance frequency of the fundamental (detector) mode induced by the an-harmonic elastic coupling between the two. Read out of the fundamental mode frequency shift signal is achieved via the AFM-type slope detection technique. In this method, the steady state amplitude of the fundamental mode is recorded at a constant carrier frequency $f_{c}$ slightly off-resonance, see Fig. 2(b). A shift in the center frequency of the fundamental peak results in a proportional change in amplitude of the resonator vibration at $f_{c}$, which is recorded using a spectrum analyzer. The sensitivity of this method is enhanced by the high quality factor $Q$ which also limits the measurement bandwidth to roughly half the width of the resonance peak.

Modal coupling is a perturbative second-order nonlinear elastic effect that can be analyzed via a Lagrangian averaging method. ${ }^{16}$ Our analysis ${ }^{13}$ predicts that the frequency shift of the fundamental mode $\Delta \omega_{1}=-c_{11} a_{1}^{2}-c_{12} a_{2}^{2}$ can be induced in two ways: (1) by forcing the mode amplitude $a_{1}$ above the linear response regime and (2) by exciting the collective mode amplitude $a_{2}$ that is coupled to it anharmonically through the 
(a)

\section{Fundamental Mode Collective Mode}

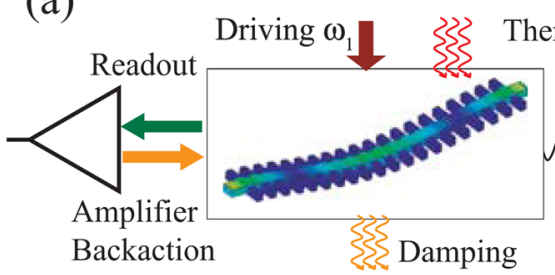

(b)

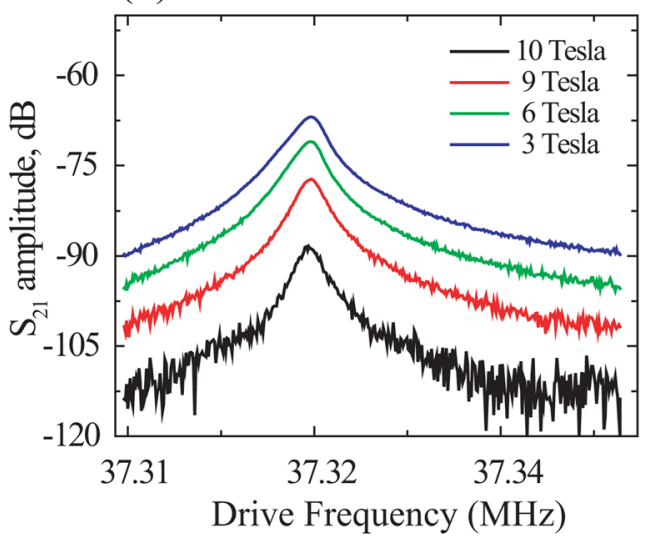

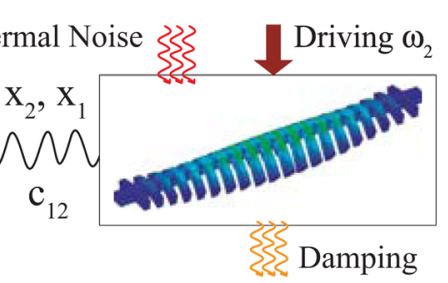

(c)

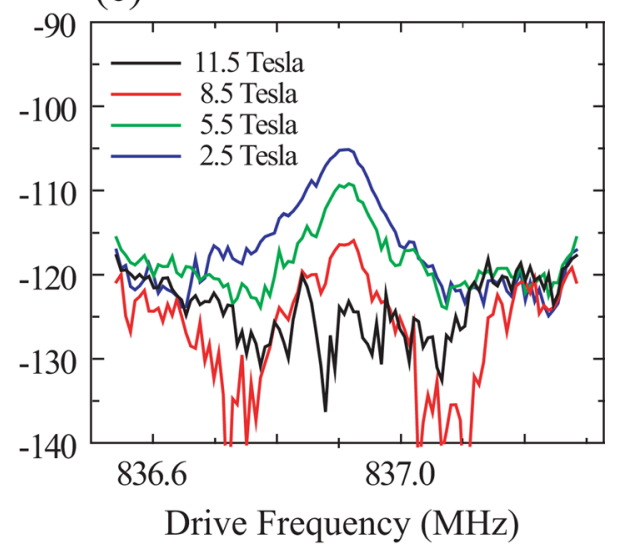

FIG. 1. (Color online) The coupled-element flexural micro-resonator with mode shapes and resonance measurements. (a) Diagram of the experimental setup involving a high frequency collective mode and a low frequency fundamental, with nonlinear elastic coupling between them. The insets show the simulated mode shapes. (b) The fundamental transverse mechanical resonance of the resonator was measured at 37.3 $\mathrm{MHz}$ with quality factor 13500 , using magneto-motive actuation and detection. (c) The collective resonance mode at $837 \mathrm{MHz}$ with quality factor 1075 . (d) SEM micrograph of a typical ultra-nanocrystalline diamond resonator device.

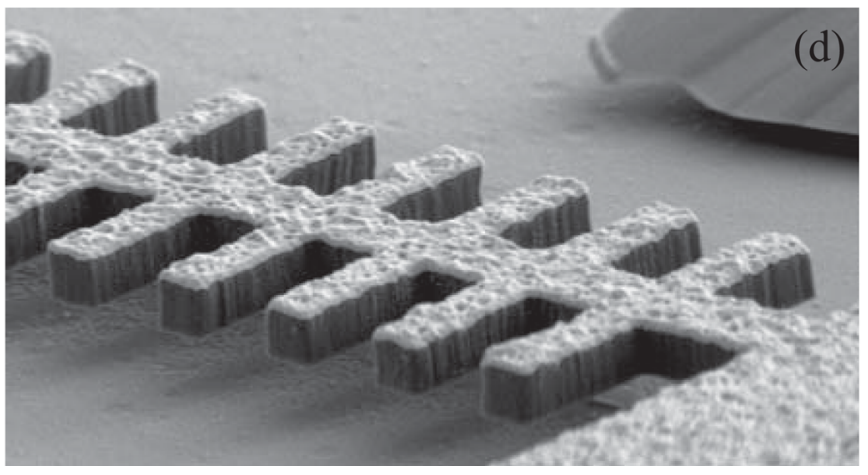

d)

$\mathrm{N} / \mathrm{m}^{3}$, see Ref. 13. The negative sign indicates that nonlinear frequency softening was modeled, while frequency hardening was observed. Both effects are common and have been observed previously, Ref. 17. Using the latter method, modal coupling can produce frequency shifts in the detector mode in $c_{12}$ coefficient. The former method produces nonlinear bi-stability and bifurcation, ${ }^{17}$ see Fig. 2(a), and we fit the response to extract the (scaled by the modal mass $M_{1}$ ) nonlinear coefficient $C_{11}=4 c_{11} M_{1}=2.15 \times 10^{13} \mathrm{~N} / \mathrm{m}^{3}$. This value agrees in magnitude with the analytical calculation of $-5.09 \times 10^{13}$

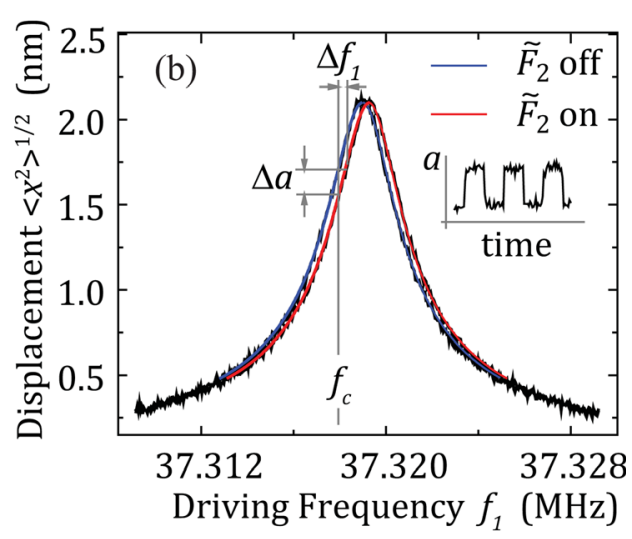

FIG. 2. (Color online) Two methods for inducing modal frequency shift. (a) Plot of the fundamental mode resonance illustrating center frequency shift at large amplitudes and the onset of nonlinear response regime. The peaks are normalized to the signal transmission background. The fitted curves are perturbation solutions to the Duffing equation that describes the response close to the resonance peak. (b) The frequency shift on the fundamental mode can also be induced by actuating it in the linear regime with constant forcing amplitude and applying a second excitation on the collective mode resonance. The plot shows the initial and the shifted peaks. The smooth curves are fitted analytical solutions, see Ref. 13. The diagram on the plot illustrates the standard AFM-type slope detection scheme for measuring the frequency shift. The inset shows amplitude modulation of the carrier frequency, which is proportional to the frequency shift at small drives. 


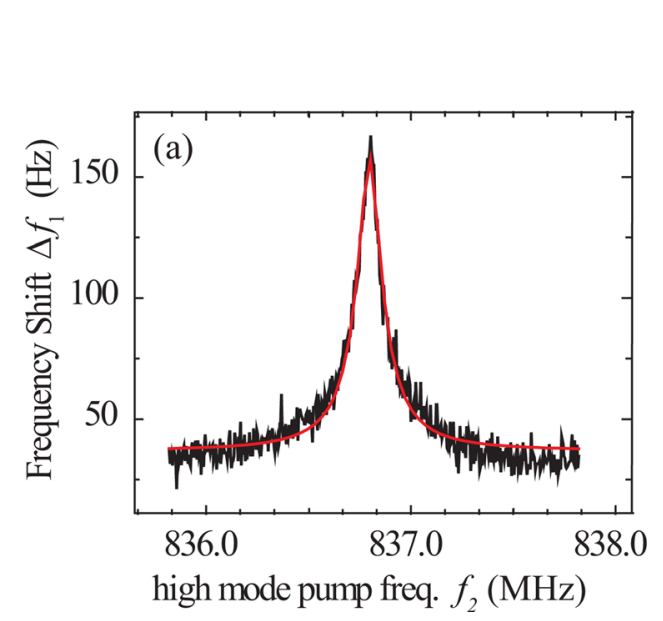

response to changes in the excitation of the collective mode, see Fig. 2(b). In this interaction, the shift in center frequency of the fundamental mode is proportional to the square of the excitation amplitude $a_{2}$ of the collective mode via the modal coupling parameter $c_{12}$. Importantly, the mechanism works even when both modes are excited well within the linear regime, far from inducing nonlinear instability in either mode. ${ }^{18}$ We extract the experimental value of the modal coupling coefficient $C_{12}=4 c_{12} M_{1}=6.06 \times 10^{18} \mathrm{~N} / \mathrm{m}^{3}$, which compares well with the analytical estimate of $1.66 \times 10^{17} \mathrm{~N} / \mathrm{m}^{3}$, Ref. 13 . In Fig. 3(a), we plot the frequency shift of the detector mode versus the collective mode excitation frequency. The detuning is proportional to the square of the collective mode amplitude, giving immediate measure of the collective mode average excitation energy.

The minimum achievable excitation level of the collective mode in our current experimental setup, see Fig. 3(b), is measured at approximately $1 \times 10^{-21}$ joules or approximately $1700 h f_{2}$ quanta of excitation energy in the collective mode at the lowest achieved temperatures of $40 \mathrm{mK}$. The limit is set by the $0.5 \mathrm{nV} / \sqrt{\mathrm{Hz}}$ input noise of the first stage preamplifier in our read-out line through the inevitable back-action effect. With future implementation of a demonstrated ultra-low noise SET (single electron transistor) detector ${ }^{5}$ or other sensitive techniques, ${ }^{19}$ we estimate two orders of magnitude of achievable noise reduction to enable single quanta resolution. Furthermore, the limited bandwidth of $1.4 \mathrm{kHz}$ (determined by the quality factor of the fundamental mode) in our setup can be removed in future experiments through frequency modulation techniques. $^{20}$
FIG. 3. (Color online) Monitoring of the collective mode excitation. (a) Plot of the fundamental mode frequency shift as a function of the collective mode driving frequency, applied near the collective mode resonance. The smooth curve is a numerical fit to the data. (b) By varying the excitation of the collective mode, the frequency shift signal on the fundamental mode is monitored down to the noise floor of the experiment. The collective mode excitation force (bottom abscissa axis) is converted to equivalent average mechanical energy (top abscissa axis) and normalized by the energy quantum for the collective mode. 